Revista Sinapsis. Vol 1, No 16, junio de 2020 | ISSN 1390 - 9770

\title{
Gafas electrónicas con sensores ultrasónicos para personas no videntes
}

\section{Electronic glasses with ultrasonic sensors for blind people}

\section{Gafas electrónicas para personas invidentes}

Kirenia Maldonado Zuñiga. ${ }^{(1)}$

Alberto Jesús Villacreses Córdova. ${ }^{(2)}$

Raquel Vera Velázquez. ${ }^{(3)}$

Martha Irene Romero Castro. ${ }^{(4)}$

(1) Universidad Estatal del Sur de Manabí, Jipijapa, Ecuador. email: kirenia.maldonado@unesum.edu.ec, ORCID: https://orcid.org/0000-0002-3764-5633

(2) Universidad Estatal del Sur de Manabí. Jipijapa, Ecuador. email: alberto1994@ @otmail.com

(3) Universidad Estatal del Sur de Manabí. Jipijapa, Ecuador. email: vera-raquel@unesum.edu.ec, ORCID: https://orcid.org/0000-0002-5071-7523

(4) Universidad Estatal del Sur de Manabí. Jipijapa, Ecuador. email: martha.romero@unesum.edu.ec, ORCID: https://orcid.org/0000-0001-5043-8295

Contacto: kirenia.maldonado@unesum.edu.ec

Recibido: 02-05-2020

Aprobado: 31-05-2020

\section{Resumen}

El objetivo de esta investigación fue desarrollar un prototipo tecnológico de gafas electrónicas que le permite a las personas no videntes a movilizarse en el entorno que los rodea, el cual les evita accidentes. La importancia de este proyecto consta en darles una calidad de vida a las personas no videntes, con el cumplimiento de los objetivos planteado se desarrolló este estratégico prototipo, en el que se detalla las funciones y característica técnicas de los diferentes componentes electrónicos que se utilizaron. Se realizó un análisis de la movilidad de las personas no videntes al momento de desplazarse de un lugar a otro, en el cual se detectaron dificultades que no les permitieron la movilidad. Se utilizaron métodos de investigación científica como, Historio-lógico, inducción-deducción, encuestas, observación y estadístico -matemático, permitieron las pautas necesarias para el proceso del estudio y el desarrollo de unas series de preguntas que se basa en encuestas y entrevistas que son primordiales para la validación de la investigación. Los resultados manifestaron los cambios significativos que se producen en la vida de las personas no videntes al utilizar las gafas electrónicas que responden a sus necesidades y disminuye los accidentes por los diferentes obstáculos, les brinda confianza para desplazarse independientemente, lo que los motiva a utilizarlas diariamente. Se concluyó con la implementación de un prototipo de gafas electrónicas con el sistema de comunicación electrónico, permite detectar obstáculos que provocan accidentes, de esta forma el usuario tiene confianza y comodidad.

Palabras clave: accidentes; discapacidad; dispositivos electrónicos; invidentes; tecnología.

\section{Summary}

The objective of this research was to develop a technological prototype of electronic glasses that allows blind people to move in the environment around them, which prevents accidents. The importance of this project consists in giving a quality of life to blind people, with the fulfillment of the objectives set, this strategic prototype was developed, which details the functions and technical characteristics of the different electronic components that were used. An analysis was made of the mobility of blind people when moving from one place to another, in which difficulties were detected that did not allow them to move. Scientific research methods such as, Historical-logical, induction-deduction, surveys, observation and statistical-mathematical were used, they allowed the necessary guidelines for the study process and the development of a series of questions based on surveys and interviews that are essential for research validation. The results showed the significant changes that occur in the lives of blind 
people when using electronic glasses that respond to their needs and reduces accidents due to different obstacles, gives them confidence to move independently, which motivates them to use them daily. It was concluded with the implementation of a prototype of electronic glasses with the electronic communication system, it allows to detect obstacles that cause accidents, in this way the user has confidence and comfort.

Keywords: accidents; disability; electronics devices; blind; technology.

\section{Introducción}

En el mundo hay unas 285 millones de personas con discapacidad visual, de las cuales 39 millones son ciegas y 246 millones presentan baja visión, según datos de la Organización Mundial para la Salud (OMS, 2011). En Latinoamérica se está desarrollando dispositivos efectivamente revolucionarios, como las gafas para personas no videntes. Las tecnologías se pueden utilizar para hacer la vida más fácil a los invidentes, crear gafas con visión artificial o sonidos que les avisen cuando están cerca de un obstáculo, ayuda a las personas con problemas de visión a realizar varias actividades cotidianas sin presentar dificultades.

Mundialmente se desarrollan diferentes dispositivos electrónicos para las personas no videntes, Latinoamérica también se une a este proyecto desarrollando dispositivos electrónicos, como las gafas para personas no videntes, México es uno de los países que realiza esta práctica, la idea es convertir los tradicionales lentes en un servicio de navegación que permitirá a las personas que las use moverse libremente, evitando en el camino tanto obstáculos como objetos en movimientos.

La investigación "Smart Guide", es el impulso en desarrollo de unas gafas inteligentes la cual ayudara a personas no videntes en su vida cotidiana, los investigadores explican que ayudan al usuario en la orientación que pueda llegar a un destino determinado gracia a la tecnología que tiene implementada (Alvarado, 2013).

La ceguera se refiere a condiciones caracterizadas por una limitación total o muy seria de la función visual, es la pérdida total física que imposibilita a la persona a desenvolverse y desarrollarse social, cultural, y económicamente solo, según el estado de perdida de la vista se pude decir que hay dos tipos de ceguera, así como hay la ceguera parcial y la ceguera total (Carreño, \& Narváez 2016).
La ceguera en cierto caso es una discapacidad con la cual se nace, pero también hay enfermedades que al no ser tratadas a tiempo llevan a la pérdida general de la visión. En los principales daños más frecuentes que limitan a la vista y llevan a la ceguera son, (Lascos-Arenas \& Valldeperas -Belmonte 2013).

A nivel nacional se han llevado a cabo las investigaciones tecnológicas para personas no videntes, la investigación a las prestaciones tecnológicas del mundo, se ha logrado realizar un sistema de orientación y movilidad diseñado para no videntes, se diseñó un sistema de sensores ultrasónico, que van ensamblada en un armazón de gafas, estratégicamente en cumplir un rango terminado las cuales enviaran señales de aviso cuando este cerca de un obstáculo (Zuffi, 2013).

Los autores, (Espinoza, \& Peña 2015) señalan resultados favorables en una investigación, en el uso de diferentes tecnologías que da soporte a las personas invidentes, que radica en ver el apoyo a las personas no vidente, mostrando un prototipo de gafas electrónicas alertando a las personas no videntes, indicando la distancia en que se encuentre un obstáculo mediante un celular. Se muestra un modelo de gafas electrónicas que advierte al celular mediante vía bluetooth capaz de advertir el acercamiento de obstáculo.

Granados \& Ortega (2015), afirman que en el proyecto de titulación, implementación de un dispositivo de gafas que ayudan a las personas no videntes, los autores afirman que el diseño de un dispositivo ultrasónico inteligente de gafas, permitirá detectar objeto, evitando que sufran golpes. Dando como resultado establecer un prototipo inteligente que enviara señales que son codificados con sonido, vibraciones dependiendo la cercanía del obstáculo.

En el estudio realizado se pudo conocer los diferentes componentes tecnológicos más importantes para enfocarse en realizar la implementación de un sistema de orientación para las personas no videntes. Se desarrolla mediante la construcción de una placa de hardware libre, el prototipo electrónico opera mediante el uso de sensores la cual transmite información sobre los diferentes obstáculos permitiendo orientarse, lo cual evitar accidentes, proporcionando mejora en la calidad de vida de los no videntes.

El objetivo del presente artículo, fue desarrollar un prototipo tecnológico de gafas electrónicas que le permite a las personas no videntes a movilizarse en el entorno que los rodea, el cual les evita accidentes. 


\section{Materiales y métodos}

La siguiente investigación se realizó en la Universidad Estatal del Sur de Manabí, a través de la técnica encuesta, a personas no videntes, estudiantes y docentes de las carreras de Ingeniería en Computación y Redes, de la población total se tomó una muestra de 138 sujetos que formaron parte del estudio y fueron encuestados.

En el estudio se realizó con la construcción de una placa de hardware libre, con el prototipo electrónico que opera mediante el uso de sensores la cual transmite información sobre los diferentes obstáculos permitiendo orientarse, lo cual evitar accidentes, proporcionando mejora en la calidad de vida de los no videntes.

Los métodos y técnicas utilizados justificaron el diagnóstico respecto a las necesidades de utilizar las gafas electrónicas. Se aplicaron entrevistas y encuestas a docentes, estudiantes y personas no videntes. La factibilidad de la investigación se comprobó a través de la aplicación informática Arduino el funcionamiento del código en la comunicación del prototipo de gafas electrónicas para detección de los diferentes objetos.

El tipo de investigación fue cualitativa, se establecieron marcos teóricos para el argumento de la problemática, cuantitativa porque a partir del estudio se centra en un análisis de los objetivos, obteniendo valores estadísticos tomados de las encuestas dirigidas a los estudiantes y entrevistas a docentes y a personas no videntes.

Se utilizó el método científico histórico-lógico en la búsqueda de los antecedentes de la investigación, que permitió conocer las necesidades de las personas no videntes a la hora de trasladarse de un sitio a otro sin ayuda de otras personas, así como las herramientas necesarias para la creación del prototipo de gafas.

Se usó el método inducción para buscar la falencia del problema de investigación ostentado en el proyecto partiendo como es la dificultad de movilizarse partir de un principio general como es la perdida de la vista, mediante este método se alcanzará observar los fenómenos o las situaciones que suceden dentro del ámbito, brindando una solución oportuna al tema de investigación.

El método deducción, facilitó el comienzo de lo general como es la discapacidad de las personas no videntes, en efecto a lo particular expresando como es la necesidad de movilizarse en el entorno, con este método se desarrollará un diagnóstico para tomar la decisión correcta sobre la implementación de las gafas electrónicas.

La observación permitió analizar la mayor cantidad de información, dando las pautas necesarias para el desarrollo del dispositivo tecnológico propuesto. Las encuestas permitieron analizar y determinar las necesidades más comunes del usuario al momento de utilizar las gafas electrónicas y las entrevistas brindaron soluciones significativas al desconocimiento que tienen muchos usuarios en el tema.

Se utilizó el método estadístico-matemático el cual permitió al investigador analizar y tabular los datos estadísticos reales en base al criterio de cada persona que participo en las encuestas, lo que permitió el desarrollo de la investigación, al conocer el estado real del problema, permite la búsqueda para la solución, esta investigación aporta un una herramienta tecnológica para las personas no vidente.

\section{Tecnologías innovadoras}

En investigaciones realizadas se ha notificado avances en estudios relacionados en impulsar el desarrollo de dispositivos que permitan ayudar a las personar no videntes, jugando un papel importante la creación de nuevas tecnologías innovadoras. En los escenarios educativos se aprecian las destrezas que obtienen los estudiantes en su formación profesional. La dificultad que presentan las personas no videntes en entorno abiertos, limita en realizar actividades de un sitio a otro, es uno de los motivos más habitual en el sentido de la perdida de la visión y deteriora su calidad de vida.

En este sentido es común que los invidentes recurran al apoyo de una persona o bastón en ciertos casos para realizar actividades o tareas, se ha demostrado que tienen problemas para la movilizarse como peatón, teniendo muchas dificultades al no presentir los obstáculos como letreros, escaleras, ramas de árboles o cualquier impedimento llegando a estar en la parte de frente de persona, ocasionando molestia incluso heridas, de esta manera se le es muy difícil al trasladarse en el medio.

Es evidente la utilización de diferentes elementos electrónicos se busca implementar un prototipo de orientación para personas no videntes, que enfrentan obstáculos en su entorno de manera que restringe en su desplazamiento. La investigación favorecerá a los invidentes, brindando así la comodidad y confianza 
necesaria en el desplazamiento, además, con la finalidad de lograr en la investigación, el desarrollo de nuevos dispositivos de ayuda, que superen las limitaciones en el medio, facilitando las actividades diarias.

Los componentes electrónicos, están conformados por una resina sintética, cerámica, metálica o plástico. Es diñado para ser conectado entre ellos, comúnmente con soldadura a un circuito impreso en cuyo paquete, de forma que los componentes emergen en una lista de dispositivo que conforma un circuito, tal manera que los elementos surgen en el desarrollo matemático en la teoría del circuito.

Un semiconductor es un componente que tiene como comportamiento guía o aislante dependiendo en varios factores a los que sea sometido, con la temperatura del ambiente, el campo electrónico o magnético al que sea expuesto entre otros. Los semiconductores son pieza importante al añadir en un circuito electrónico, con ellos se cuenta con diversos componentes tales como diodos, transistores entre otros (Ramírez, 2016).

Los sensores es aquel componente que convierte magnitudes físicas en valores medibles de dicha magnitud, puedes ser de temperatura, presión, caudal del agua, objetos entre otros, para adquirir un conocimiento de cantidades físicas que, por naturaleza o tamaño, no se consigue ser percibida directamente por los sentidos de la persona, para los movimientos de orientación sea por las caracteriza propias o al contrario de circuitos o dispositivos intermedios (Ramírez, 2016).

Según Iglesias \& Lago (2014), la batería es un componente que convierte la energía química a energía eléctrica mediante reacción de forma de cargas, la batería se puede recargar varias veces, dado así no están atribuido en la segunda ley termodinámica siendo enérgicamente más eficientes. La unidad de la batería conforma de una o varias celdas que son conectadas en forma de serie o paralelo, en relación a las especificaciones de salida y la cabida de recarga almacenado de energía deseada.

Las gafas electrónicas, son un dispositivo que va montado en la cabeza, conformada por un sistema electrónico y sensores ultrasonido, puede detectar obstáculos tales como ramas letreros, arbusto u otro obstáculo. Las personas no videntes reciben información sobre el entorno, mediante sonidos o vibraciones, pueden ser guiados, evadiendo obstáculos en el medio que los rodea a una distancia de ochenta $\mathrm{cm}$.

El sistema de las gafas electrónicas, consta de un módulo de hardware libre como arduino y sensor de ultrasónico que ayudan a prevenir obstáculos a personas no videntes, mediante el aviso de vibraciones y sonido, por un módulo de carga para la batería de esta manera da conocer diferentes componentes electrónicos, demostrando el tipo de funcionamiento que da el microcontrolador al dispositivo, y el manejo y uso de los sensores ultrasónicos que es fundamental en el funcionamiento del dispositivo, cuyo propósito es demostrar que la investigación ayuda al fortalecimiento en desarrollar de este nuevo dispositivos.

El dispositivo de las gafas electrónicas en las personas no videntes, teniendo el dispositivo dos partes en su estructura, la primera parte en la cabeza y otra en el abdomen, el equipo cumple la función de orientar al usuario no vidente al caminar. Las gafas electrónicas permiten la detección de los diferentes obstáculos, primordialmente aquellos objetos que obstaculizan la movilidad de las personas, facilitando la movilización de las personas no videntes.

\section{Resultados}

La investigación evidencio, la importancia de conocer los diferentes componentes tecnológicos más importantes en la implementación de un sistema de orientación para las personas no videntes. Se desarrolla mediante la construcción de una placa de hardware libre, y el prototipo electrónico opera mediante el uso de sensores la cual transmite información sobre los diferentes obstáculos permitiendo orientarse, evitar accidentes, proporcionando una mejora en la calidad de vida a los no videntes.

En lo relacionado a la encuesta aplicada a 138 sujetos, conformada por personas no videntes, estudiantes y docentes de las carreras de Ingeniería en Computación y Redes, de la Universidad Estatal del Sur de Manabí, se logró evidenciar los siguientes resultados que se observan en el gráfico 1. El 86\% de los educandos en formación afirman, no tener conocimientos de los dispositivos de guía, mientras el $14 \%$ si tienen conocimiento. 


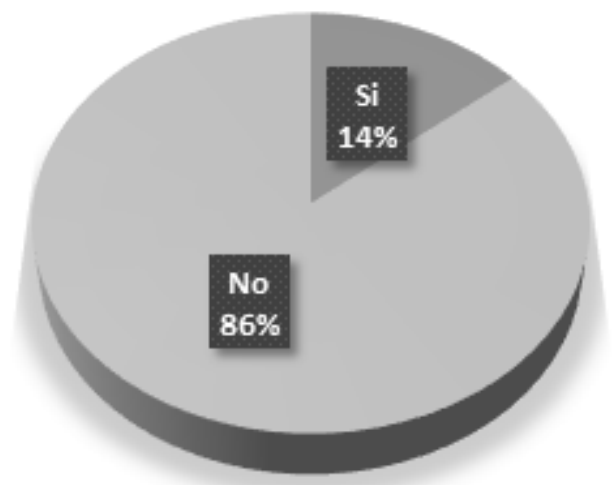

Gráfico 1. ¿Tiénes conocimientos del dispositivo electrónico de guía para personas no videntes?

$\mathrm{Al}$ realizar la pregunta para indagar en lo referente a los conocimientos de los educandos en relación a los componentes electrónicos, se pudo conocer que el $20 \%$, afirma que tiene conocimiento, mientras que el
$80 \%$ afirman no conocer los componentes para el diseño de un prototipo de gafas electrónicas, se observa en el gráfico 2.

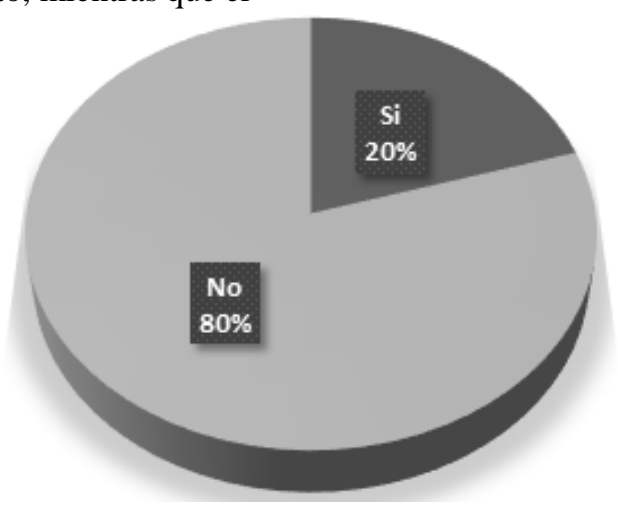

Gráfico 2. ¿Conoce usted los componentes electrónicos para el diseño de las gafas?

En cuanto a la otra pregunta, así conocen el tipo de programación del módulo de arduino, se pudo conocer que el $63 \%$ de los estudiantes no tienen conocimiento en el tipo de programación, mientras que el 37\% afirman en saber el tipo de lenguaje que usa. La mayoría de los estudiantes plantean que no tienen conocimiento en la programación que usan los módulos arduinos.

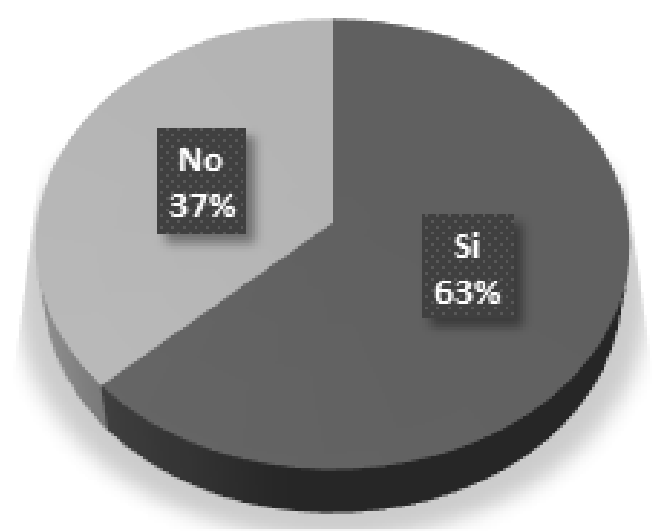

Gráfico 3. ¿Tiénes conocimiento del tipo de programación del módulo de arduino? 
En el gráfico 4 , los resultados revelan que el $46 \%$ de los estudiantes tienen conocimiento, mientras el $54 \%$ afirman no tener conocimiento en el manejo de los sensores ultrasónico. Lo que demuestra las carencias de saberes en este tema en la mayoría de los estudiantes que participaron en el estudio.

En cuanto a la factibilidad al usar los sensores ultrasónicos, el $83 \%$ de los estudiantes afirman la posibilidad de usarlos, mientras que un $17 \%$ comenta que no tienen noción. Los resultados revelan que el uso de los sensores ultrasónicos es una pieza fundamental en las gafas electrónicas, de manera que cumple la función de alertar para prevenir los obstáculos en beneficio de las personas no videntes, se observa en el gráfico 5

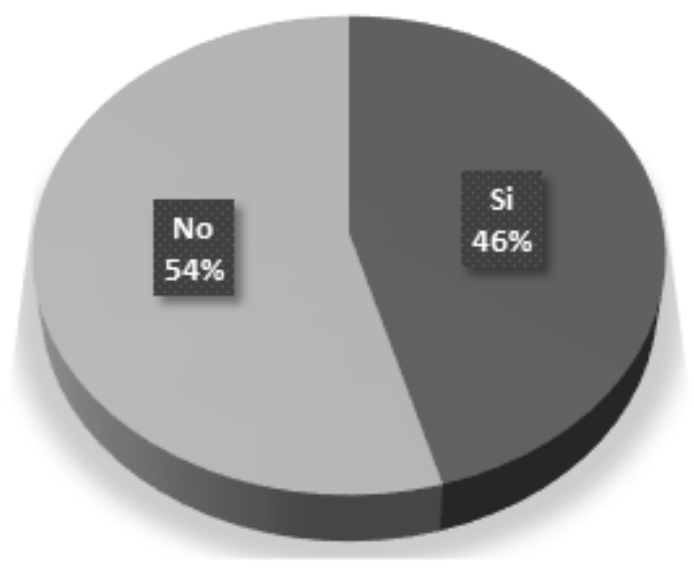

Gráfico 4. ¿Tiéne usted conocimiento sobre el uso y manejo de los sensores ultrasónicos?

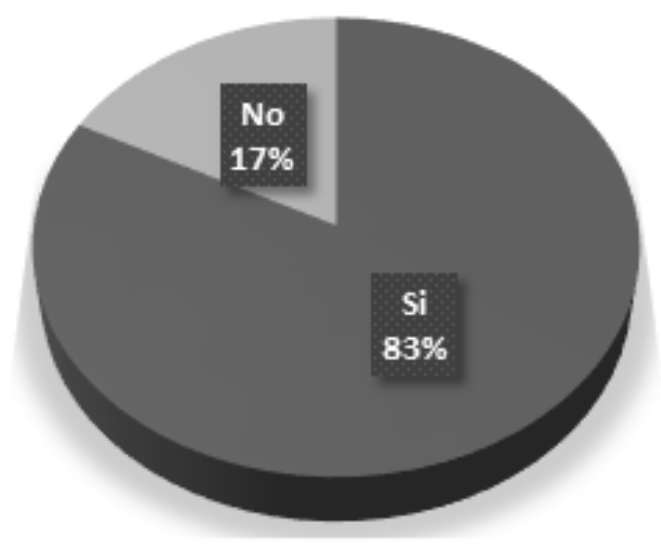

Gráfico 5. ¿Considera usted, que el uso de sensores en las gafas es fundamental para prevenir los obstáculos?

En lo concerniente al gráfico 6, se puede apreciar que al realizar la pregunta relacionada así es necesario diseñar un prototipo de gafas electrónicas para personas no videntes en el laboratorio de robótica, se observa que el $80 \%$ de los estudiantes afirman que es importante, mientras el $20 \%$ demostraron que no. 


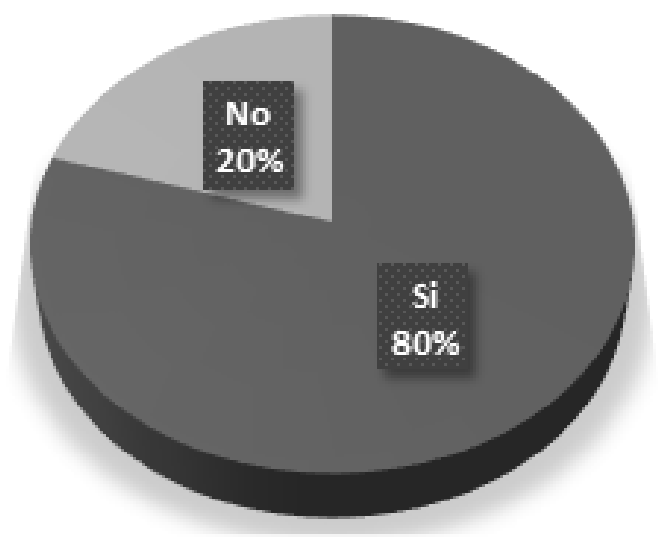

Gráfico 6. Personas no videntes, estudiantes y docentes de las carreras de Ingeniería en Computación y Redes, de la Universidad Estatal del Sur de Manabí. Elaborado por: Autores

\section{Discusión}

En esta experiencia se coincide con Loayza \& Constante (2016), que relatan la importancia de los dispositivos de detección de obstáculos para las personas no videntes, con el propósito de que los alerten ante cualquier peligro. En este estudio se concuerda con otras investigaciones realizadas por otros autores que afirman haber creado un dispositivo de navegación y detección de obstáculos para las personas no videntes (Lengua, Dunai, Peris, \& Defez 2013)

En otras investigaciones efectuadas por Obando, Taramuel \& Hugo, Víctor (2015), relatan haber desarrollado un sistema electrónico de gafas, para ayudar a la orientación de personas no videntes, que se basa en un dispositivo con sensores ultrasónicos, percíbela presencia o ausencia de un obstáculo. Obteniendo resultados positivos para transitar las personas no videntes, en esta investigación se concuerda con estos autores.

La programación arduino es importante porque permite traducir códigos y poder interactuar con el medio exterior la persona no vidente, con la presencia de sensores ultrasónicos. Se concuerda con los autores Santo-Oncero (2007), relatan que la programación de arduino, es la programación de un microcontrolador. Por otra parte programar arduino consiste en traducir a líneas de código las tareas automatizadas que queremos hacer leyendo de los sensores y en función de las condiciones del entorno programar la interacción con el mundo exterior mediante unos actuadores.

Se coincide con otras investigaciones, en lo relacionado a los sensores ultrasónicos son detectores de proximidad que trabajan libres de roces mecánicos y que detectan objetos a distancia que van desde pocos $\mathrm{cm}$ hasta 4 metros (Taramuel, \& Gonzalo 2015). El sensor emite un sonido y mide el tiempo que la señal tarda en regresar.

El uso de sensores en las gafas es fundamental para prevenir los obstáculos en las personas no videntes según los autores Molina \& LLanga (2015), en su proyecto estudio de equipos que ayudan a las personas no videntes, demuestran la finalidad de conocer el diseño de un equipo mediante el uso de sensores ultrasónicos de proximidad, creando un prototipo de gafas que lleva puesto el no vidente, dando así la localización de obstáculos con servicio de comando de alerta de vos de manera que el usuario podrá llevar un mejor desenvolvimiento en su entorno.

El dispositivo transmisor y el receptor están en parte separada sobre el propio sensor, haciendo de este más exacto, dado que la distancia de detección mínima es menor a las vibraciones emitida por el emisor, afectando en menor proporción la señal recibida, lo que expresa que la frecuencia de repetición es mayor, al 25 y $250 \mathrm{~Hz}$, muy importante el estudio de estos autores que analizaron de forma convincente en el "diseñó de un equipo de gafas que ayude a las personas invidentes al desplazarse" el empleo de un dispositivo de detección de obstáculos, alertando al usuario que se detenga o continúe caminando, de esta manera los dispositivos serán más accesibles para los invidentes, proporcionara opciones que mejoraran el entorno de sus vidas habitual (Loayza \& Constante 2016)

Aquino, \& Martínez (2016), manifiestan que las instituciones educativas superior tienen como 
compromiso en facilitar guías a los estudiantes no videntes, es de gran importancia fortalecer el apoyo educativo requerido para su guía en los establecimientos educativos, llevando acabo normativas en la trayectoria de la investigación, lo cual se debe promover la accesibilidad en aquellos estudiantes que padezca con esta discapacidad de perdida de la vista, mejorando el desempeño de aprendizaje en las instituciones educativas.

Según Escudero, \& Sotomayor (2016), en su investigación plantean que las personas no videntes son más propensa al tener accidentes en sus hogares, la dificulta que tienen las personas ciegas al no poder circular en su casa, de esta manera es dar una solución con el desarrollar de dispositivos que tenga la capacidad de advertir al usuario de impedimentos físicos cercanos así ellos, dando la alternativa al usuario de movilizarse de un lugar a otro sin llegar al tener heridas

\section{Conclusiones}

En la investigación se analizaron las características técnicas de los componentes electrónicos, para la construcción del prototipo de gafas electrónicas, orientado a las personas no videntes. Se desarrolló el sistema electrónico, cumpliendo la función de envío y recepción de datos que emiten los sensores, para la comunicación al usuario en la detección de los diferentes obstáculos. Se contribuyó en la orientación y movilidad a las personas no videntes. Se realizaron las debidas pruebas del dispositivo con las personas no videntes, donde se demuestra el funcionamiento esperado de las gafas electrónicas en las diferentes posiciones donde se llegará a tener un impedimento al transitar, tanto en la parte superior del torso como la parte del abdomen, el sistema responde cuando un objeto está al frente de ellos, comunicando al usuario mediante el sonido y vibración. El uso del dispositivo en las personas no videntes cumple con un rango de $80 \mathrm{~cm}$, para detectar los obstáculos.

\section{Bibliografía}

Alvarado, R. (2013). Tecnologia Innovadora Para Personas No Videntes. Tecnologia de Mexico, 1- 25.

Aquino, S. \& Martínez, V. (2016). La inclusión educativa de ciegos y baja visión en el nivel superior. Un estudio de caso. Lima: Sinectica Educativa.
Carreño, J. , \& Narváez, J. (2016). Angles en el fútbol, colonía vacacional para niños de 10 a 16 años con capacidades diferentes no videntes en la ciudad de Cuenca. Bachelor's thesis, 1-71.

Espinoza, D., \& Peña, C. (2015). Diseño e implementación de un prototipo de gafas electrónicas con comunicación bluetooth a un celular para la detección de objetos circundantes que servirá como ayuda para personas no videntes. Guayaquil: Universidad Politécnica Salesiana Ecuador.

Escudero, K. \& Sotomayor, N. (2016). Prototipo de gafas electronicas para no videntes. Cuenca: Escuela Politécnica Nacional.

Granados, D. \& Orteg, J. (2015). Dispositivo ultrasónico inteligente para personas con deficiencia visual. México: Instituto Politécnico Nacional.

Iglesias, R., \& Lago, A.( 2014). Modelado y Simulación de una Batería de Ion-Litio Comercial Multicelda. Departamento de Tecnología Electrónica, Universidad de Vigo, 11-17.

Loayza, M. \& Constante, P. (2016). Investigación de un sistema electrónico con detección de obstáculos para personas no videntes. Latacunga: Universidad de las Fuerzas Armadas ESPE Extensión Latacunga. Recuperado de http://repositorio.espe.edu.ec/xmlui/bitstream /handle/21000/11886/ESPEL-MEC-0081$\underline{\text { P.pdf? }}$ sequence $=2$ \&isAllowed $=\mathrm{y}$

Lascos-Arenas, J., \& Valldeperas -Belmonte, X. (2013). Cirugía del glaucoma mediante esclerectomía profunda no perforante con implante supraciliar. Universitat Autónoma de Barcelona, 1-154.

Lengua, I.; Dunai, L.; Fajarnes, G.P.; Defez, B. (2013). Navigation device for blind people based on Time-of-Flight technology. DYNA, 80, 33-41.Recuperado de http://www.scielo.org.co/pdf/dyna/v80n17 9/v80n179a04.pdf

Molina, A. \& Llanga, A. (2015). Diseño e implementación de un prototipo de gafas electrónicas para personas no videntes. Riobamba: Universidad Nacional de 
Chimborazo.Recuperado

de

http://dspace.unach.edu.ec/bitstream/51000 /12/1/UNACH-EC-IET-2015-0001.pdf.pdf

Taramuel, V. \& Gonzalo, N. (2015). Diseño y construcción de un sistema de orientación para ayuda en la movilidad de personas invidentes. Quito: Escuela Politécnica Nacional.Recuperado de https://bibdigital.epn.edu.ec/bitstream/1500 0/9045/4/CD-6034.pdf

OMS. (2015). 285 millones de personas con discapacidad visual según la OMS. Recuperado https://www.paho.org/uru/index.php?optio $\mathrm{n}=\mathrm{com}$ content $\&$ view $=$ article $\& \mathrm{id}=451: 285$ -millones-personas-discapacidad-visual$\underline{\text { segun-oms \&Itemid=247 }}$

Ramirez, G. (2016). Diseño e implementación de un sistema para detección y monitoreo de la concentración de gas GLP mediante arreglo de sensores orientado al sector residencial.

Santo-Oncero, D.(2007).Hardware libre.Todo linux, Madrid :p 21-12.

Zuffi, R. C. (2013). Traje tecnológico para no videntes. Electrónica Conocimiento , 39-52. 